\section{Chapple v. Ganger}

Robert L. Heilbronner

Chicago Neuropsychology Group, Chicago, IL, USA

\section{Historical Background}

In the case of Daubert v. Merrell Dow (1993), it was ruled that for scientific testimony to be admissible, it has to be (a) scientifically valid and (b) relevant to the case at hand. The court provided a list of guidelines intended to aid in the determination of scientific validity (e.g., peer reviewed, falsifiability, acceptable error rate, etc.). The Daubert ruling along with subsequent related rulings (e.g., General Electric v. Joiner 1997; Kumho Tire v. Carmichael 1999) generated significant debate among psychologists and neuropsychologists and many other disciplines. Specifically, Reed (1996) viewed the Daubert ruling to necessitate the utilization of commercially available fixed batteries only, such as the Halstead-Reitan Battery. However, most neuropsychologists employ a flexible battery approach; thus, contradicting Reed's assertions implying that most neuropsychologists would not be suited for involvement in forensic work. In support of his conclusion, Reed referenced the case of Chapple v. Ganger (1998), a brain injury claim. Review of the judge's written decision in Chapple v. Ganger outlined that all neuropsychological testimony (even partial HRB protocols from two other neuropsychologists) was admitted into evidence and the fact that the judge had placed more emphasis on testimony from a fixed battery advocate was completely unrelated to the determination of the test battery. Indeed, there was no Daubert challenge to a flexible test battery approach. In this particular case, only the testimony of a vocational specialist who conducted no testing and provided no evidence in the form of a peer-reviewed study to support his claims was subject to a Daubert hearing from the defense.

\section{References and Readings}

Chapplev. Ganger, 851 F. Supp. 1481, E.D. of Washington (1994).

Daubert v. Merrell Dow Pharmaceuticals, 509 U.S. 579 (1993).

General Electric Co. v. Joiner, 522 U.S. 136 (1997).

Greiffenstein, M. F., \& Cohen, L. (2005). Neuropsychology and the law: Principles of productive attorneyneuropsychologists relations. In G. Larrabee (Ed.), Forensic neuropsychology: A scientific approach. New York: Oxford University Press.

Kumho Tire Co. v. Carmichael, 526 U.S. 137 (1999).

Reed, J. E. (1996). Fixed versus flexible neuropsychological test batteries under the Daubert standard for the admissibility of scientific evidence. Behavioral Sciences and the Law, 14, 315-322. 